\title{
The modulation of inhibition of return by object- internal structure: Implications for theories of object-based attentional selection
}

\author{
IRENE REPPA and E. CHARLES LEEK \\ University of Wales, Bangor, Wales
}

\begin{abstract}
Recently, Vecera, Behrmann, and McGoldrick (2000), using a divided-attention task, reported that targets are detected more accurately when they occur on the same structural part of an object, suggesting that attention can be directed toward object-internal features. We present converging evidence using the object-based inhibition of return (IOR) paradigm as an implicit measure of selection. The results show that IOR is attenuated when cues and targets appear on the same part of an object relative to when they are separated by a part boundary. These findings suggest that object-based mechanisms of selection can operate over shape representations that make explicit information about objectinternal structure.
\end{abstract}

There is now considerable evidence that attentional selection may be directed toward locations (e.g., B. A. Eriksen \& C. W. Eriksen, 1974; C. W. Eriksen \& St. James, 1986; Posner, 1980) or toward particular objects in the environment (e.g., Baylis \& Driver, 1992; Driver \& Baylis, 1989; Duncan, 1984; Kramer \& Jacobson, 1991; Lavie \& Driver, 1996; Tipper, Driver, \& Weaver, 1991; Vecera, Behrmann, \& Filapek, 2001; Vecera, Behrmann, \& McGoldrick, 2000; Vecera \& Farah, 1994).

One empirical effect that has been used to elucidate the nature of location- and object-based selection is inhibition of return, or IOR (e.g., Klein, 2000; Posner \& Cohen, 1984). Using a spatial cuing paradigm, Posner and Cohen found that participants took longer to detect targets presented at the same spatial location as that where a peripheral cue had been presented than to detect targets presented at uncued locations. Subsequent studies have shown that inhibition is not restricted solely to previously cued spatial locations, but also to previously cued objects (e.g., Abrams \& Dobkin, 1994; Gibson \& Egeth, 1994; Jordan \& Tipper, 1999; Leek, Reppa, \& Tipper, 2003; Tipper et al., 1991). For example, Tipper et al. (1991) examined IOR using apparent motion displays, in which cues and targets could appear at either the same or different spatial locations within the same perceptual object (a square). Their results showed that IOR was still observed (although attenuated) when cues and targets appeared within the same perceptual object, even

This work was supported by Grant R000239512from the Economic and Social Research Council (U.K.) to E.C.L. and by a postgraduate fellowship from Unilever PLC to I.R. We thank Patricia Reuter-Lorenz, Tram Neill, and Luis Fuentes for their helpful comments on an earlier version of the manuscript. Correspondence should be addressed to I. Reppa, Centre for Cognitive Neuroscience, University of Wales (Bangor), The Brigantia Building, Penrallt Road, Gwynedd LL592AS, U.K. (e-mail: i.reppa@bangor.ac.uk). though the spatial location of the object had changed between cue offset and target onset.

These studies demonstrate that attention may be directed toward objects, yet relatively little is known about the kinds of shape representations that mediate objectbased selection. With regard to this issue, hypotheses about shape representation in the domain of visual object recognition may provide important constraints on theories of object-based attention (e.g., Vecera et al., 2001; Vecera et al., 2000).

Several different proposals about the content and structure of shape representations have been advanced. One broad distinction that has been made concerns the representation of global versus local feature structure (e.g., Navon, 1977; Palmer, 1977). Some accounts of object recognition emphasize global shape properties such as those derived from occluding or outline contour (e.g., Haywood, 1998). According to other hypotheses, recognition is assumed to be critically mediated by representations containing information about local (object-internal) feature structure (e.g., Biederman, 1987; Hoffman \& Richards, 1984; Leek \& Arguin, 2000; Marr, 1982; Palmer, 1977; Pentland, 1989). Among variants of the latter class of hypothesis are differing views about the kinds of shape primitives that represent object structure (e.g., Biederman, 1987; Leek \& Arguin, 2000; Marr, 1982), the nature of part boundaries (e.g., Biederman, 1987; Hoffman \& Richards, 1984; Hoffman \& Singh, 1997), and the way in which information about the spatial configuration of shape features is encoded (e.g., Biederman, 1987; Marr, 1982).

Of particular relevance to the present paper is that this broad distinction between global and local shape features can be used to motivate competing hypotheses about the nature of the shape representations mediating objectbased attention. One possibility is that object-based se- 
lection operates over representations that contain information only about global shape properties. Some evidence in support of this hypothesis comes from a recent study of grouping effects in negative priming by Fuentes, Humphreys, Agis, Carmona, and Catena (1998). They showed that grouping letters (one target and two distractors) by surrounding them with a box in a prime display attenuates inhibition (negative priming) for the same distractors presented on following trials in a probe display. This finding suggests that object-based attention may select objects as grouped whole forms (i.e., the box with the letters) without necessarily making explicit their internal structure (the letters within the box).

Another possibility is that object-based selection operates over locally structured shape representationsthat is, over representations that make explicit internal shape features such as part boundaries. ${ }^{1}$ Some support for this hypothesis, at least in the domain of facilitation, comes from a recent experiment by Vecera et al. (2000; see also Vecera et al., 2001). Using a divided-attention task, Vecera et al. (2000) found that participants were more accurate in reporting different features from the same part of an object than in reporting features belonging to different parts of the object, suggesting that facilitatory attentional modulation can be constrained by object-internal shape properties. However, one limitation of these studies is that they rely on an explicit measure of the distribution of attention across object structure; arguably, the task (to report features belonging to specific shape parts) may bias selection toward relevant object features. ${ }^{2}$

In this study, we addressed these issues by providing converging evidence using an implicit measure of attentional selection. The rationale is based on findings that inhibition (Gibson \& Egeth, 1994; Jordan \& Tipper, 1999; Tipper et al., 1991), as well as facilitation (Egly, Driver, \& Rafal, 1994; Moore, Yantis, \& Vaughan, 1998), can spread along the surface of a cued object. Jordan and Tipper examined object-based inhibition in an IOR paradigm. Using displays of two rectangles (Egly et al., 1994), participants were required to respond to the onset of a target (white square) that could appear following the brief presentation of a peripheral cue at a location within one of the two objects in the stimulus displays. Responses were slower to targets presented at uncued locations on the surface of a previously cued object than to targets appearing at equidistant locations on uncued objects, demonstrating object-based inhibition. In the present study, we examined whether the magnitude of this IOR effect would be modulated by object-internal shape features, such as an internal structural discontinuity (or part boundary) in a cued object that occurs in between a cue and a target location. If IOR operates over internally structured shape representations, then the magnitude of IOR may be modulated by internal structural features of objects that are selected for attention. In contrast, if selection operates solely over global shape descriptions that lack internal part boundaries, then object-internal structure would not be expected to modulate IOR. We examined these predictions in two experiments exploiting object-based IOR. In Experiment 1, we replicated the object-based inhibition effect for 2-D stimulus displays reported by Jordan and Tipper. In Experiment 2, we examined whether the magnitude of IOR is modulated by the presence of object-internal structural discontinuities in the stimulus displays.

\section{EXPERIMENT 1}

The purpose of Experiment 1 was to replicate the object-based IOR effect found by Jordan and Tipper (1999) and to establish a set of task parameters that would reliably produce object-based IOR. The prediction was that if inhibitory modulation by attention can be object based, then IOR should occur at uncued locations on the surface of previously cued objects.

\section{Method}

Participants. Twenty undergraduates from the University of Wales, Bangor, participated in the experiment. They all reported normal or corrected-to-normal vision. Each participant received one course credit.

Apparatus and Stimuli. Stimuli were presented on a 14-in. monitor connected to a Power Macintosh PC. Randomization and presentation of the stimuli, as well as recording of the participants' reaction times (RTs), were controlled through PsyScope software (Version 1.2.4). Responses were made through a single letter key on a standard Apple keyboard connected to the computer.

The stimulus display consisted of two outline (black) rectangles, simultaneously presented on each side of a fixation cross (Figure 1) against a light gray background. The rectangles subtended $1.5^{\circ} \times$ $6.5^{\circ}$ of visual angle when viewed from a $55-\mathrm{cm}$ distance. The orientation of the rectangles varied randomly between trials, appearing at $+45^{\circ}$ or $-45^{\circ}$ tilted from the vertical meridian on $50 \%$ of each of the trials. The fixation cross (+) subtended $0.8^{\circ} \times 0.8^{\circ}$ of visual angle. The cue was an outline white square subtending $1.0^{\circ} \times 1.0^{\circ}$, with contours subtending $0.02^{\circ}$. The target was a filled white square measuring $0.8^{\circ} \times 0.8^{\circ}$. The central refixation cue was a white cross (+) subtending $0.8^{\circ} \times 0.8^{\circ}$. Stimulus displays subtended a total of $9.7^{\circ}$ of visual angle vertically and $9.7^{\circ}$ horizontally.

Design. A 3 (cue-target location) $\times 3$ (stimulus onset asynchrony [SOA]) within-subjects design was used. The three cue-target locations are shown in Figure 2. In Condition 1-same object/same location - the target appeared on the same object and at the same location as did the cue. In Condition 2-same object/different location-the target appeared on the same object but at a different location from the cue's. In Condition 3-different object/baselinethe target appeared on a different object from the cue, but at a location corresponding to the location probed in the cued object.

Additional filler trials (different object/filler) were also included. In these trials the target appeared on a different object from the cue and at a location diagonal to the cue (Figure 2). In Conditions 2 and 3, the spatial distance between cue-target locations was identical $\left(5.2^{\circ}\right.$ of visual angle). In the filler trials, cue-target distance exceeded $5.2^{\circ}$ (data from these trials were therefore excluded from the analyses; see below).

The second within-subjects factor was SOA, with three levels: 400,820 , and $1,220 \mathrm{msec}$. There were 100 trials in each cue-target location condition, distributed across the three SOAs. Trials were presented randomly across cue-target location condition and SOA. Participants completed 10 practice trials followed by 600 experimental trials; 200 of these were no-target trials. The ratio of target to no-target trials was $3: 1$. 


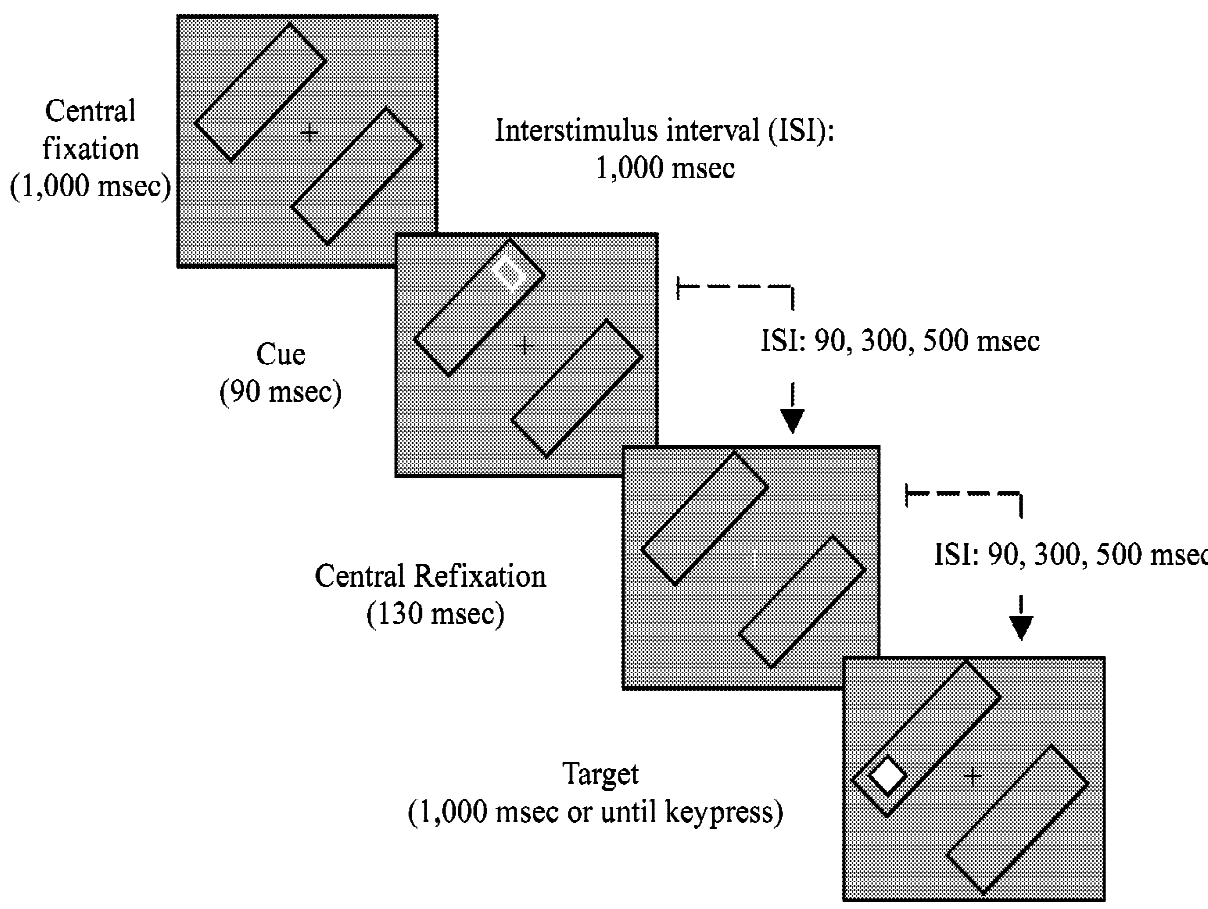

Figure 1. An illustration of a same object/different location trial when the rectangles were presented at $+\mathbf{4 5}^{\circ}$ orientation. The target was presented within the same object but at a different location from the cue.

Procedure. The trial procedure is shown in Figure 1. At the beginning of each trial, a fixation cross was shown in the center of the monitor. After $1,000 \mathrm{msec}$, two rectangles were simultaneously presented on each side of the fixation cross. Following a further 1,000msec delay the peripheral cue appeared at one of four locations at either end of the two stimulus rectangles. Cue location was random and equiprobable. Cue duration was $90 \mathrm{msec}$. At intervals of 90 , 300 , or $500 \mathrm{msec}$ from cue offset, the central fixation cross changed from black to white for a period of $130 \mathrm{msec}$ and then reverted to black until the end of the trial (central refixation). After a further delay of 90,300 , or $500 \mathrm{msec}$, the target was presented at one of the four possible cue locations. The target remained visible for $1000 \mathrm{msec}$, or until the "b" key (response) was depressed.

Participants were informed that the white outline square (the cue) was not predictive of the location of the subsequent target. They were instructed to press the response key as soon as they detected the target and to withhold their response when no target was presented. If they failed to do so, a $500-\mathrm{Hz}$ tone was generated by the computer, and the trial was considered an error. The importance of fixating the cross was stressed during the practice period and just before the experiment commenced.

\section{Results and Discussion}

Mean correct RTs from target-present trials in all four cue-target location conditions were calculated. All trials with RTs that were less or greater than $3 S D$ s from the mean RT per condition, as well as incorrect responses (responses to no-target trials), were discarded from the data. This accounted for $1.5 \%$ of all trials. RTs for all four cue-target location conditions are reported in Table 1.

Further analyses were performed only on data from the three cue-target location conditions, in which the distance between cues and targets was identical (same object/same location, same object/different location, and different object/baseline). This was essential in order to avoid confounds of space- and object-based effects-that is, differences in the magnitude of inhibition between conditions that might arise solely because of variation in cue-target proximity (e.g., Jordan \& Tipper, 1999).

The key issue in Experiment 1 was whether IOR is obtained even when cues and targets appeared at different locations on the cued object. That is, if IOR affected only previously cued spatial locations, and not objects, then there should be no time cost in detecting targets that appeared at different spatial locations on previously cued objects. In order to examine this, IOR was measured by subtracting mean RTs in the two cued-object conditions (same object/same location and same object/different location) from the mean RTs in the different object/ baseline condition. Figure 3 shows the magnitude of mean object-based IOR effects in the cued-object conditions as a function of SOA.

RTs from the three cue-target location conditions were analyzed using a 3 (cue-target location: same object/same location, same object/different location, and different object/baseline) $\times 3$ (SOA: 400, 820, and 1,220 msec) repeated measures analysis of variance (ANOVA). There were significant main effects of cue-target location $[F(2,38)=124, p<.001]$ and SOA $[F(2,38)=5.0, p<$ $.012]$, with the slowest RTs at an SOA of $1,220 \mathrm{msec}$ $(M=408, S D=31.5)$. The interaction between cue-target 


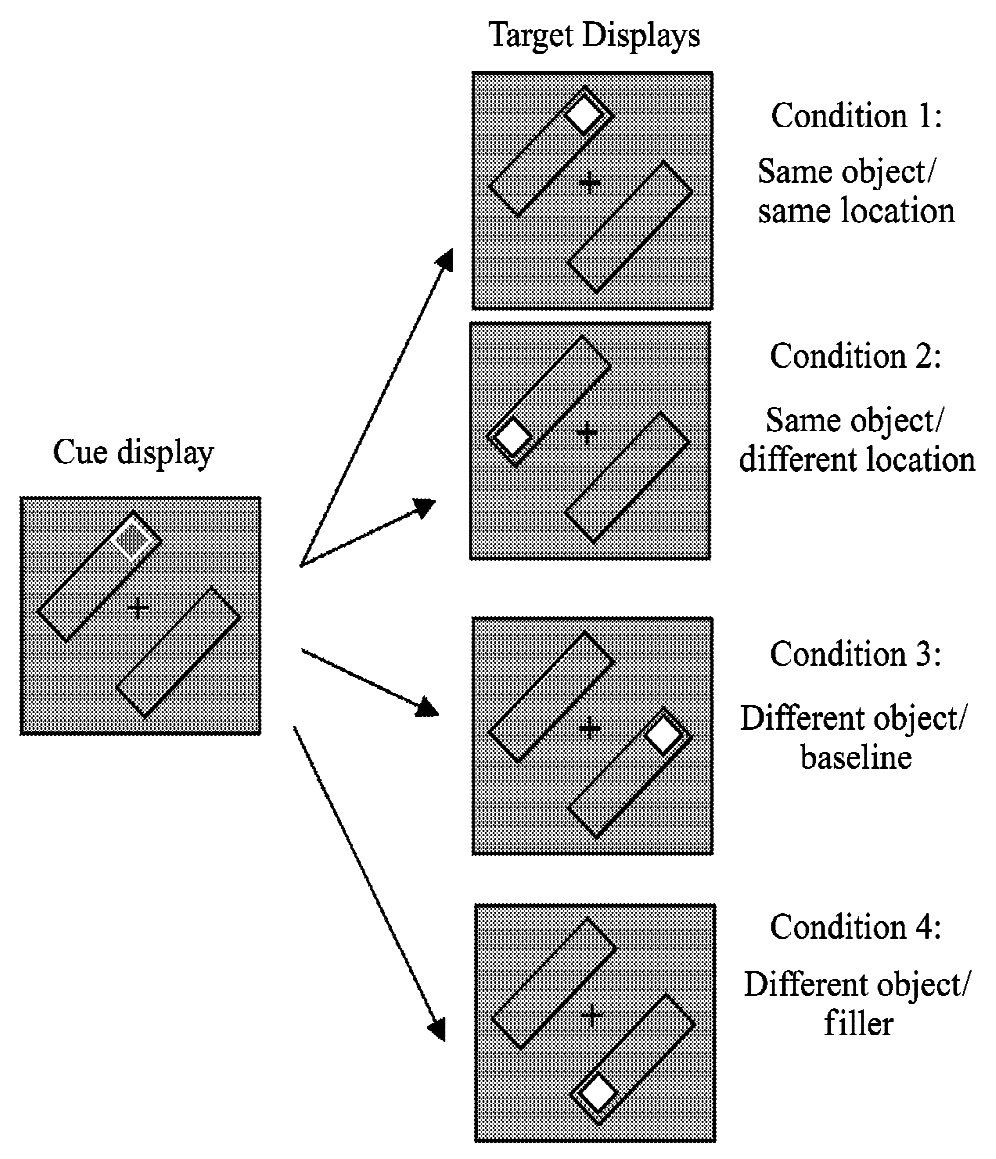

Figure 2. Illustration of the four (two within-object and two between-object) cue-target location conditions in Experiment 1.

location and SOA was not significant $[F(4,76)=0.83$, n.s.]. Planned comparisons were carried out for RTs in each cue-target location condition (collapsed over SOA). These showed a significant IOR effect for the same object/same location condition collapsed over SOA of $36 \mathrm{msec}[t(19)=-13.9, p<.001]$ and a significant IOR effect of $11 \mathrm{msec}$ for the same object/different location condition $[t(19)=-4.0, p<.01]$.

The results of Experiment 1 confirmed Jordan and Tipper's (1999) findings that object-based IOR can spread across the surface of a 2-D object and provided us with a set of experimental parameters that might be used to examine whether IOR is modulated by object-internal structural features. We examined this question in Experiment 2.

\section{EXPERIMENT 2}

The aim of Experiment 2 was to investigate whether object-internal structure modulates object-based IOR.

Table 1

Mean RTs (in Milliseconds) for the Four Cue-Target Location Conditions (Including the Filler Condition) at Each SOA

\begin{tabular}{|c|c|c|c|c|}
\hline & Condition 1 & Condition 2 & Condition 3 & Condition 4 \\
\hline SOA & $\begin{array}{l}\text { Same Object/ } \\
\text { Same Location }\end{array}$ & $\begin{array}{c}\text { Same Object/ } \\
\text { Different Location }\end{array}$ & $\begin{array}{c}\text { Different Object/ } \\
\text { Baseline }\end{array}$ & $\begin{array}{c}\text { Different Object/ } \\
\text { Filler } \\
\end{array}$ \\
\hline 400 & 404 & 375 & 363 & 359 \\
\hline 820 & 396 & 373 & 364 & 355 \\
\hline 1,220 & 408 & 385 & 374 & 367 \\
\hline
\end{tabular}

Note-Calculation of IOR resulted by subtracting RTs in the different object/baseline condition from each of the two within-object conditions (same object/same location and same object/different location). Mean RTs from the different object/filler condition (Condition 4) were not used in the analysis. 

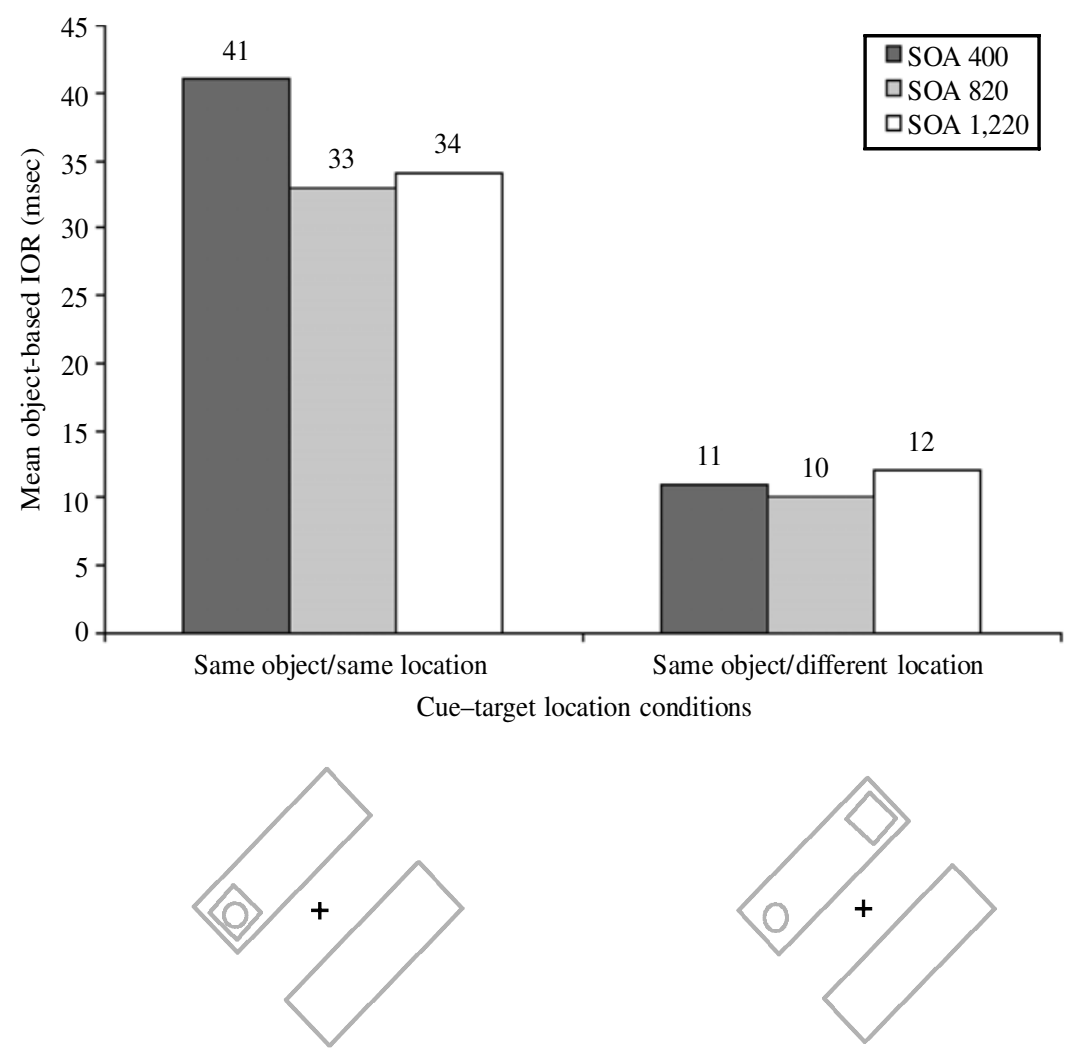

Figure 3. Object-based IOR effect for the two within-object cue-target location conditions grouped by SOA. For purposes of illustration, cues are depicted as circles and targets as squares. In the same object/same location condition, targets appear within the same location as the cue following a time interval.

This issue was examined using stimulus displays in which cues and targets were presented on 2-D L-shaped forms that were either segmented by an internal structural discontinuity (a single edge contour) or unsegmented (Figure 4). Otherwise, the task was identical to that of Experiment 1. The predictions were as follows: If object-based IOR operates over internally structured shape representations, then the spread of IOR may be modulated by the structural discontinuity in the segmented shapes when the cue and target are presented on different sides of the discontinuity (i.e., on different object parts). In contrast, if selection operates over representations that contain solely global shape properties (e.g., outline shape), then the magnitude of object-based IOR should be equivalent between segmented and unsegmented object displays.

\section{Method}

Participants. Ten participants, registered as psychology majors at the University of Wales, Bangor, took part in the experiment, each receiving one course credit. All reported normal or correctedto-normal vision. None had participated in Experiment 1.

Apparatus and Stimuli. The same apparatus as in Experiment 1 was used. Displays consisted of two outline L shapes consisting of a long arm and a short arm (or rectangle) appearing on either side of a fixation cross at the center of the screen. There were two types of stimulus displays (see Figure 4). In the segmented displays, both L shapes contained an internal line segment (or discontinuity) separating the long and short arms of the figure. In the unsegmented displays, there was no internal line segment between the long and short arms.

In all trials, the $\mathrm{L}$ shapes were tilted to the right $\left(+45^{\circ}\right)$. In half of the trials, the short arms of the L shapes appeared above and below fixation (Figure 4, left column); in the other half, the short arms appeared on the left and on the right of fixation (Figure 4, right column).

At a viewing distance of $50 \mathrm{~cm}$, the longer arm of each $\mathrm{L}$ shape subtended $7.2^{\circ} \times 1.8^{\circ}$ of visual angle, and the shorter arm $2.8^{\circ} \times$ $2.2^{\circ}$. The black fixation cross subtended $0.8^{\circ} \times 0.8^{\circ}$. The cue $(\mathrm{a}$ white outline square) subtended $0.6^{\circ} \times 0.6^{\circ}$ (contours measuring $0.2^{\circ} \times 0.2^{\circ}$ ), and the target (a filled white square) $0.8^{\circ} \times 0.8^{\circ}$. The whole display subtended approximately $13.2^{\circ} \times 10.8^{\circ}$ of visual angle.

Design. A 4 (cue-target location) $\times 2$ (object: segmented vs. unsegmented) $\times 2$ (SOA: 820 and 1,220 msec) within-subjects design was used. The four cue-target conditions for both types of object are shown in Figure 4. In all experimental trials (59\% of all trials), cues were presented in the middle of the long arm of the cued object. Filler trials using other cue locations were also included. Cue-target locations in the experimental conditions for both segmented and unsegmented displays were as follows: In Condition 1, 


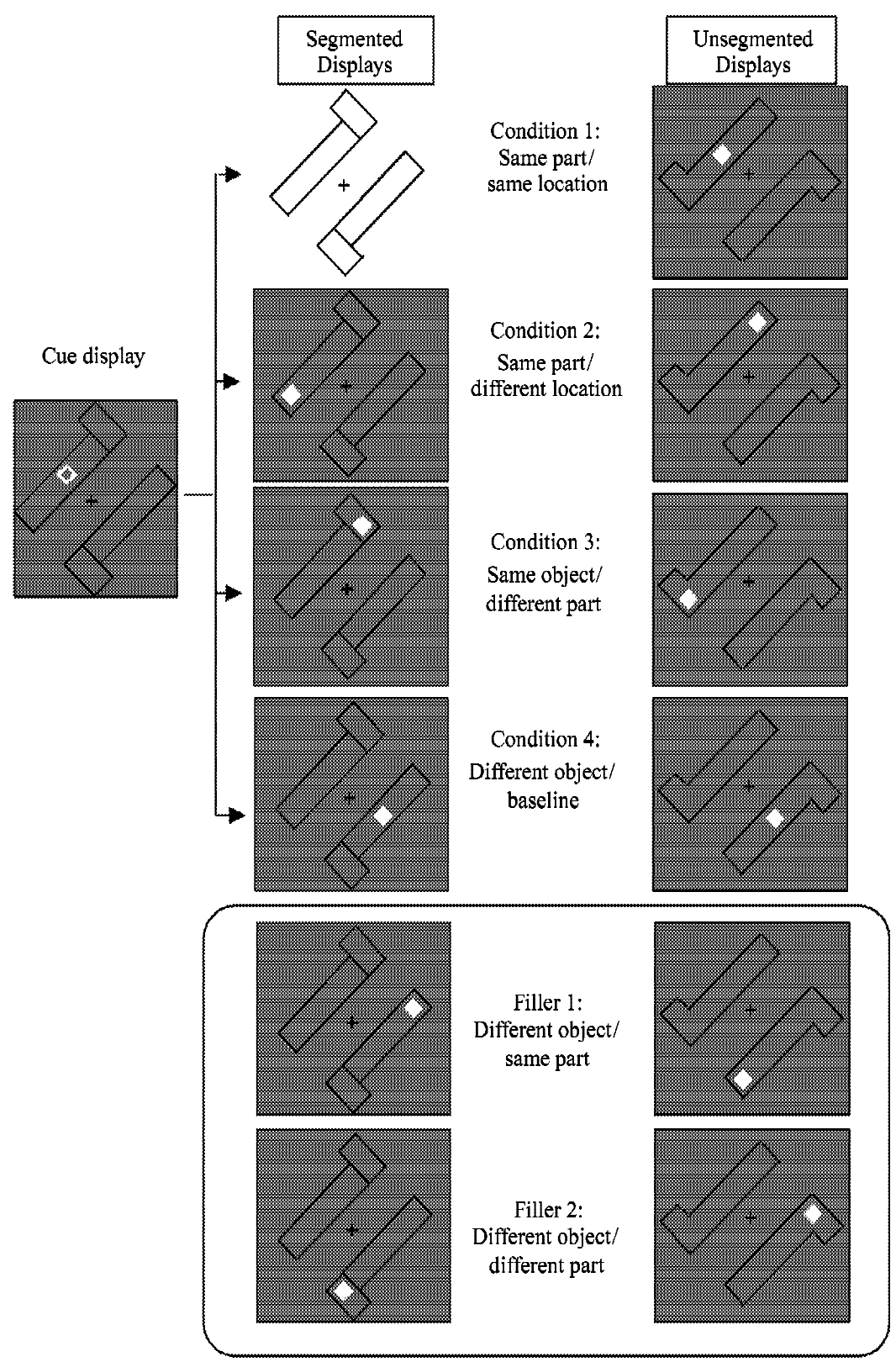

Figure 4. Six possible cue-target location conditions when the cue appeared at the center of one of the $L$ shapes. The two columns illustrate the six possible target locations for the segmented (left column) and unsegmented (right column) displays. Filler conditions are depicted in the lower panel but were not analyzed, because the cue-target distance was greater than the cue-target distance in any of the within-object cue-target location conditions. Procedure was the same as in Experiment 1.

same part/same location, the target appeared on the same segmented part and at the same location as did the cue. In Condition 2, same part/different location, the target appeared on the same segmented part as did the cue but at a different location. In Condition 3, same object/different part, the target appeared on the same object but on a different part than did the cue. In Condition 4, different object / baseline, the target appeared on the middle of the longer arm in the uncued object (i.e., at the same corresponding location as the cue). This condition was used as the baseline measure for IOR because the distance between cues and targets (cue-target proximity) was identical to that in Conditions 2 and 3 ( $4.7^{\circ}$ of visual angle). Thus, this condition permitted calculation of object-based IOR effects across conditions in which cue-target proximity was held constant (Jordan \& Tipper, 1999). 
There were also two types of different-object filler trials involving other cue-target locations (Figure 4, lower panel). These were not equated in terms of cue-target proximity. On these trials, targets always appeared on the uncued object, either on the part corresponding to the cued location (Figure 4, Filler 1) or on a different part (Figure 4, Filler 2). Cue-target distance in the filler trials was not equated to cue-target distance in Conditions $2-4$. These trials were excluded from the analysis because of the spatial confound of cue-target proximity.

Each participant completed 10 practice trials followed by 680 experimental trials, of which $200(30 \%)$ were no-target trials. In the remaining 480 target trials, there were 80 trials in each of the six (including the filler trials) cue-target location conditions. In each cue-target location condition there were 40 trials with segmented object displays and 40 trials with unsegmented object displays collapsed across the two SOAs. ${ }^{3}$

Procedure. The procedure in Experiment 2 was identical to that of Experiment 1.

\section{Results and Discussion}

All trials with RTs that were less or greater than $3 S D$ s from the mean RT per condition, as well as incorrect responses, were discarded from the data. This accounted for $1.2 \%$ of all trials. Mean RTs for Conditions 1-4 (same part/same location, same part/different location, same object/different part, different object/baseline), along with mean RTs for the two different-object filler conditions, are shown in Table 2.

The aim of the analyses was to determine whether the magnitude of object-based IOR is modulated by the presence of an object-internal discontinuity between the cue and target-in other words, whether the size of the IOR effect is different between equivalentcue-target locations in the segmented and unsegmented displays. In order to examine this question, IOR was measured by subtracting mean RTs in the three same-object cue-target location conditions (1, 2, and 3$)$ from the mean RTs in the different object/baseline condition (4), collapsed across SOA. As noted above, only these conditions were included in the analysis because cue-target proximity was identical, allowing us to obtain a measure of changes in the magnitude of object-based IOR independently of the distance between cues and targets (Jordan \& Tipper, 1999). Figure 5 shows the magnitude of mean IOR effects in each condition for segmented and unsegmented displays.
Mean RTs were analyzed using a 4 (cue-target location) $\times 2$ (object) $\times 2(\mathrm{SOA})$ repeated measures ANOVA. There was a significant main effect of cue-target location $[F(3,27)=14.7, p<.001]$. There were no significant main effects of object or SOA $[F(1,9)=0.03$, n.s., and $F(3,27)=0.4$, n.s., respectively]. The interaction between cue-target location and object was significant $[F(3,27)=2.0, p<.05]$.

Separate planned comparisons were carried out for the magnitude of IOR between Conditions 2 and 3 (same part/different location and same object/different part). For the segmented object displays, IOR was significantly larger when the cue and target locations were separated by the internal discontinuity than when both cue and target appeared on the same part; that is, IOR was larger in Condition 3 than in Condition $2[t(9)=3.42, p<.001]$. In contrast, for the unsegmented object displays, there was no significant difference in the magnitude of IOR between the two conditions $[t(9)=0.62, p>.05]$.

Planned comparisons between the segmented and the unsegmented object conditions showed that IOR for the segmented displays in the same part/same location conditions was significantly larger than that in the unsegmented displays (42 vs. $31 \mathrm{msec}$, respectively) $[t(9)=$ $3.07, p<.05]$. In addition, the difference in IOR between segmented and unsegmented displays for the same object/different part condition was also significant (42 vs. $21 \mathrm{msec}$, respectively) $[t(9)=4.01, p<.05]{ }^{4}$

In order to rule out the possibility that the difference between the two critical cuing conditions (same part/ different location and same object/different part) could be due solely to low-level visual factors, post hoc tests were carried out between the two filler cuing conditions (Figure 4). In these trials the cue appeared in the center of one of the two objects, and the target subsequently appeared on either end of the other (uncued) object. In the (uncued) segmented displays, mean RTs for Filler $1(M=$ $325 \mathrm{msec})$ were not significantly different from mean RTs for Filler $2(M=327 \mathrm{msec})[t(9)<1$, n.s.]. The difference between Filler $1(M=327 \mathrm{msec})$ and Filler 2 $(M=329 \mathrm{msec})$ in the (uncued) unsegmented displays was not significant either $[t(9)<1$, n.s. $] .^{5}$ Thus, the modulation in the time taken to detect targets appearing

Table 2

Mean RTs (in Milliseconds) for Each of the Four Experimental and Two Filler Cue-Target Location Conditions in Experiment 2

\begin{tabular}{|c|c|c|}
\hline \multirow[b]{2}{*}{ Cuing Condition } & \multicolumn{2}{|c|}{ Display Type } \\
\hline & Segmented & Unsegmented \\
\hline Condition 1: Same part/same location & 361 & 357 \\
\hline Condition 2: Same part/different location & 345 & 341 \\
\hline Condition 3: Same object/different part & 360 & 347 \\
\hline Condition 4: Different object/baseline & 318 & 326 \\
\hline Filler 1: Different object/same part & 321 & 333 \\
\hline Filler 2: Different object/different part & 324 & 332 \\
\hline
\end{tabular}

Note-Mean RTs in the Filler 1 and Filler 2 conditions are reported only for the purpose of completeness but were not used in the statistical analysis. 

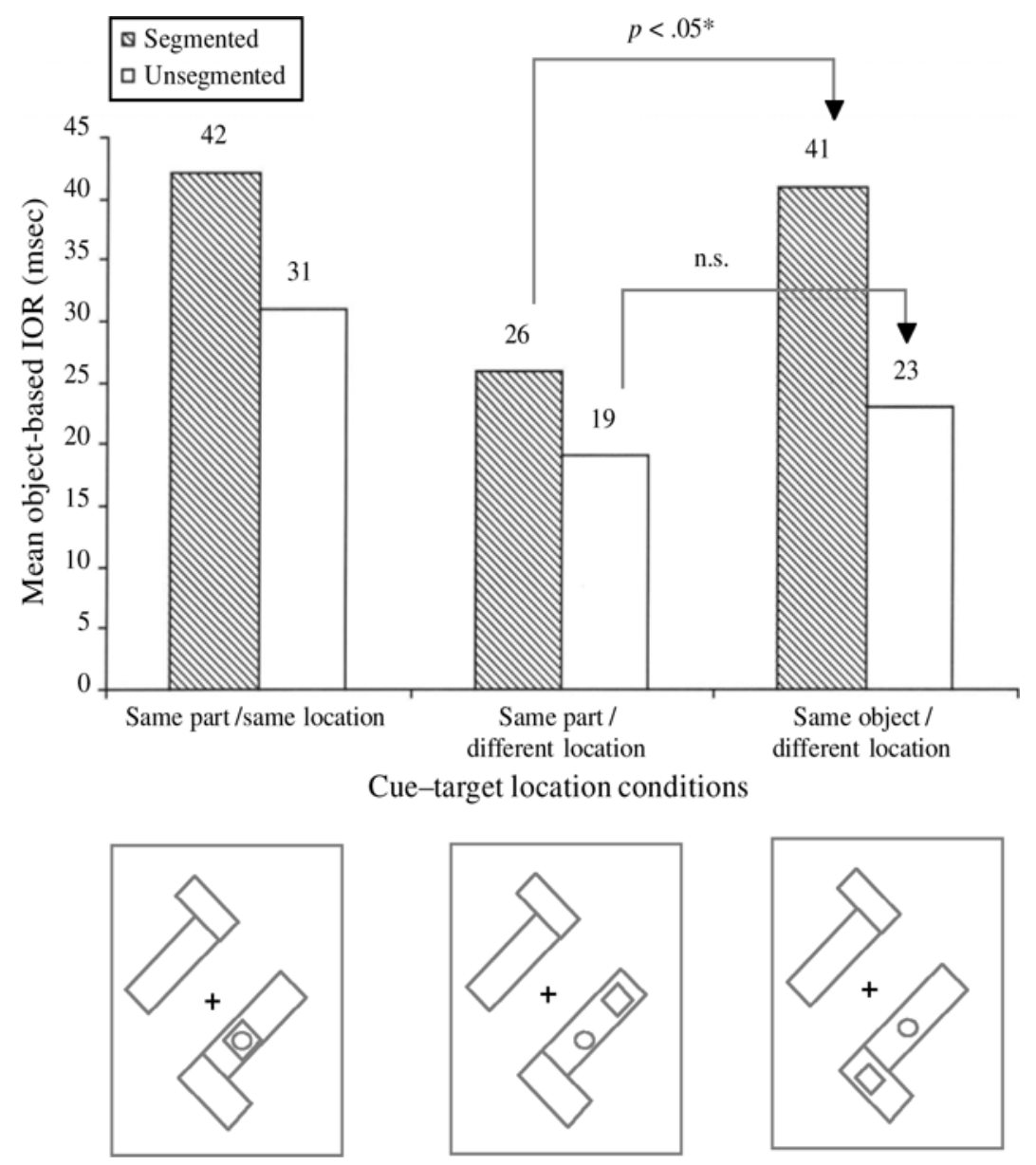

Figure 5. Object-based IOR effect for the three within-object cue-target location conditions for the segmented and unsegmented displays. The data are collapsed across the two SOAs. For illustration purposes cues are depicted as circles and targets as squares.

on the same or different object components in the segmented displays is, indeed, found only with cued displays, consistent with an IOR effect.

In summary, the main finding of Experiment 2 was that an internal structural discontinuity presented between a cue and target significantly affected the magnitude of object-based IOR. In addition, IOR was larger when the cues and targets were separated by the internal discontinuity - that is, when the cue and target appeared on different parts of the same object. The implications of these findings for hypotheses about object-based selection are discussed below.

\section{GENERAL DISCUSSION}

In summary, Experiment 1 showed that object-based IOR can generalize across a single surface of an object, replicating the object-based IOR effect reported by Jordan and Tipper (1999). Experiment 2 showed that the magnitude of object-based IOR is modulated by the presence of an object-internal structural discontinuity. In par- ticular, IOR is greater when cues and targets appear on different object parts than when they both appear on the same part.

These findings are consistent with the hypothesis that object-based attentional selection can operate over shape representations that make explicit information about object-internal structure, rather than solely across shape representations that contain only global shape properties (such as outline contour). These results converge with the findings of Vecera et al. (2000; Vecera et al., 2001) in revealing the part-based modulation of attention.

These findings also extend previous evidence in two important ways. First, unlike in the earlier studies using divided-attention tasks (Vecera et al., 2001; Vecera et al., 2000), the present data, from the IOR paradigm, show that part-based modulation of attention is also found using implicit measures of selection, even when the object (and its internal features) is irrelevant to the task. Second, the finding that IOR is greater when cues and targets are separated by a putative internal part boundary than when they both appear on the same object compo- 
nent provides a new constraint on hypotheses about the mechanisms underlying object-based selection. Indeed, were the modulation of object-based IOR based solely on the spread of inhibition across representations of global object shapes, irrespective of object-internal structure, one might assume that IOR would be greater the closer the target appeared to the cue, on the assumption that there would be a higher probability of summation of location- and object-based inhibitory effects (e.g., Gibson \& Egeth, 1994; Jordan \& Tipper, 1999). On this account, the magnitude of object-based IOR should decrease as an inverse function of (within-object) cue-target proximity. Contrary to this prediction, we found that object-based IOR increased for targets appearing on different object components even when cue-target proximity is held constant.

One possibility is that this effect reflects the differential spread of facilitation and inhibition across internal components of object shapes, in agreement with previous reports showing the simultaneous operation of facilitatory and inhibitory components of attention (e.g., MariBeffa, Houghton, Estevez, \& Fuentes, 2000; Tassinari, Aglioti, Chelazzi, Peru, \& Berlucchi, 1994; Tipper et al., 1997). For example, Mari Beffa et al., using a letter search task, found that letter-word grouping reduced negative priming effects when the task was to report a letter (target) within a word (distractor). Negative priming was found when the letter to be reported appeared outside the distractor word. Thus, targets appearing within the cued part of a task-relevant object may be subject to both facilitation, through grouping, and inhibition, depending on the cue-target interval. In contrast, targets appearing outside the cued part of an object appear to be subject only to inhibitory effects. Thus, in the present case, we might speculate that facilitation and inhibition operate in parallel during object-based selection but spread differentially across object structure. Whereas inhibition spreads equally across both cued and uncued parts, facilitation may accrue only on cued object components (or spread over both components at different rates). The summation of these different effects would predict a pattern of IOR modulation between cued and uncued object parts that is consistent with the present results: greater IOR for cues and targets appearing on different object components.

A different explanation, however, for the pattern of IOR modulation found in Experiment 2 may lie in the visual complexity of the segmented displays relative to the unsegmented displays. Indeed, IOR is significantly greater, overall, for the segmented than for the unsegmented displays, even in the same part/same location condition, where the internal discontinuity should have played little or no role. Thus, one might argue that visual complexity (perhaps defined in terms of the number of edge segments in a stimulus), as opposed to the sensitivity of object-based attention to internal structure, underlies the apparent modulation of IOR found in the segmented displays. However, this view might be challenged on at least two grounds. First, as shown in the analyses, there was no significant difference in RTs between the two (uncued object) filler conditions (when the cue appeared at the center of one of the objects and the target appeared at either end of the uncued object), despite the presence of the internal boundary in the stimulus on which the target was shown. This indicates that the modulation of object-based IOR found here is not simply an artifact of visual complexity; if it were, differences in RTs should also have been found with targets shown on uncued objects in the segmented displays. This reinforces our claim that the pattern of modulation found in Experiment 2 reflects changes in the magnitude of an IOR effect. Second, although the precise functional significance of the internal discontinuity remains unclear (e.g., whether it constitutes a part boundary per se), an interpretation of the data in terms of greater visual complexity in the segmented displays does not undermine our claim that IOR must operate over locally structured shape representations. This is because any presumed increase in visual complexity, which might modulate target detection latency in the segmented displays, must also be assumed to arise from the presence of the internal discontinuity, which, by hypothesis, implies that local shape information is made explicit in the representations mediating task performance.

Finally, the present findings also invite speculation about the structure of the shape representations that mediate visual object perception. As noted earlier, there are several competing proposals about the kinds of shape information that are encoded in these representations. These include representations based on low-level features such edges and outline contour (e.g., Lowe, 1985; Ullman, 1989), surfaces (e.g., He \& Nakayama, 1992; Leek \& Arguin, 2000; Marr \& Nishihara, 1978; Pentland, 1989), and/or volumetric shape components (e.g., Biederman, 1987; Marr \& Nishihara, 1978). Our data show that object-based selection can operate over internally structured shape representations, at least for 2-D forms, but they do not distinguish among these competing hypotheses. We are currently further investigating the role that different kinds of putative part boundaries may play in shape representation and in the modulation of object-based IOR.

In conclusion, we have shown that object-based IOR is modulated by internal features of objects. This result provides a constraint on hypotheses about the kinds of shape representations that mediate object-based selection. More specifically, they show that object-based selection does not operate over shape representations that solely contain information about global shape properties, but over representations that make explicit information about internal shape structure.

\section{REFERENCES}

Aвrams, R. A., \& Dobkin, R. S. (1994). Inhibition of return: Effects of attentional cueing on eye movement latencies. Journal of Experimental Psychology: Human Perception \& Performance, 20, 467-477.

Bay lis, G. C., \& Driver, J. (1992). Visual parsing and response competition: The effect of grouping factors. Perception \& Psychophysics, 51, 145-162. 
Biederman, I. (1987). Recognition-by-components: A theory of human image understanding. Psychological Review, 94, 115-147.

Driver, J., \& BAY LIS, G. C. (1989). Movement and visual attention: The spotlight metaphor breaks down. Journal of Experimental Psychology: Human Perception \& Performance, 15, 448-456.

DunCAN, J. (1984). Selective attention and the organization of visual information. Journal of Experimental Psychology: General, 113, 272300.

Egly, R, Driver, J., \& Rafal, R. D. (1994). Shifting visual attention between objects and locations: Evidence from normal and parietal lesion subjects. Journal of Experimental Psychology: General, 123, 161-177.

Eriksen, B. A., \& Eriksen, C. W. (1974). Effects of noise letters upon the identification of a target letter in a nonsearch task. Perception \& Psychophysics, 16, 143-149.

ERIKSEN, C. W., \& ST. JAmES, J. D. (1986). Visual attention within and around the field of focal attention: A zoom lens model. Perception \& Psychophysics, 40, 225-240.

Fuentes, L. J., Humphreys, G. W., Agis, I. F., Carmona, E., \& Catena, A. (1998). Object-based perceptual grouping affects negative priming. Journal of Experimental Psychology: Human Perception \& Performance, 24, 664-672.

Gibson, B. S., \& Egeth, H. (1994). Inhibition of return to object-based and environment-based location. Perception \& Psychophysics, 55, 323-339.

Hay Ward, W. G. (1998). Effects of outline shape in object recognition. Journal of Experimental Psychology: Human Perception \& Performance, 24, 427-440.

He, Z. J., \& Nakayama, K. (1992). Surfaces vs. features in visual search. Nature, 359, 231-233.

Hoffman, D. D., \& Richards, W. A. (1984). Parts of recognition. Cognition, 18, 65-96.

Hoffman, D. D., \& Singh, M. (1997). Salience of visual parts. Cognition, 63, 29-78.

Jordan, H., \& Tipper, S. P. (1999). Spread of inhibition across an object's surface. British Journal of Psychology, 90, 495-507.

KLEIN, R. (2000). Inhibition of return. Trends in Cognitive Sciences, 4 , 138-147.

Kramer, A. F., \& Jacobson, A. (1991). Perceptual organization and focused attention: The role of objects and proximity in visual processing. Perception \& Psychophysics, 50, 267-284.

LAVIE, N., \& Driver, J. (1996). On the spatial extent of attention in objectbased visual selection. Perception \& Psychophysics, 58, 1238-1251.

Leek, E. C., \& ARguin, M. (2000, November). Surface-based shape descriptions in object recognition: Preliminary evidence from partwhole matching and priming. Paper presented at the annual meeting of the Psychonomic Society, New Orleans.

Leek, E. C., Reppa, I., \& Tipper, S. P. (2003). Inhibition of return for objects and locations in static displays. Perception \& Psychophysics, 65, 388-395.

Lowe, D. G. (1985). Perceptual organisation and visual recognition. Boston: Kluwer-Nijhoff.

Mari-Beffa, P., Houghton, G., Estevez, A. F., \& Fuentes, L. J. (2000). Word-based grouping affects the prime-task effect on semantic priming. Journal of Experimental Psychology: Human Perception \& Performance, 26, 469-479.

MARr, D. (1982). Vision. New York: Freeman.

Marr, D., \& Nishihara, H. K. (1978). Representation and recognition of the spatial organization of three-dimensional shapes. Proceedings of the Royal Society of London: Series B, 200, 269-294.

Moore, C., Yantis, S., \& VAughan, B. (1998). Object-based visual selection: Evidence from perceptual completion. Psychological Science, 9, 104-110.

NAvon, D. (1977). Forest before the trees: The precedence of global features in visual perception. Cognitive Psychology, 9, 353-383.

Palmer, S. E. (1977). Hierarchical structure in perceptual representation. Cognitive Psychology, 9, 441-474.
Pent Land, A. (1989). Shape information from shading: A theory about human perception. Spatial Vision, 4, 165-182.

Posner, M. I. (1980). Orienting of attention. Quarterly Journal of Experimental Psychology, 32, 3-25.

Posner, M. I., \& Cohen, Y. (1984). Components of visual orienting. In H. Bouma \& D. G. Bouwhuis (Eds.), Attention and performance $X$ (pp. 531-556). Hillsdale, NJ: Erlbaum.

Tassinari, G., Aglioti, S., Chelazzi, L., Peru, A., \& Berlucchi, G. (1994). Do peripheral non-informative cues induce early facilitation of target detection? Vision Research, 34, 179-189.

Tipper, S. P., Driver, J., \& Weaver, B. (1991). Short report: Objectcentred inhibition of return of visual attention. Quarterly Journal of Experimental Psychology, 43A, 289-298.

Tipper, S. P., Rafal, R. D, Reuter-Lorenz, P. A., Starrveldt, Y., Ro, T., Egly, R., Danzinger, S., \& Weaver, B. (1997). Objectbased facilitation and inhibition from visual orienting in the human split-brain. Journal of Experimental Psychology: Human Perception \& Performance, 23, 1522-1532.

Ullman, S. (1989). Aligning pictorial descriptions: An approach to object recognition. Cognition, 32, 193-254.

Vecera, S. P., Behrmann, M., \& Filapek, J. C. (2001). Attending to the parts of a single object: Part-based selection limitations. Perception \& Psychophysics, 63, 308-321.

Vecera, S. P., Behrmann, M., \& McGoldrick, J. (2000). Selective attention to the parts of an object. Psychonomic Bulletin \& Review, 7, 301-308.

Vecera, S. P., \& Farah, M. J. (1994). Does visual attention select objects or locations? Journal of Experimental Psychology: General, 123, 146-160.

\section{NOTES}

1. Unless otherwise specified, throughout the paper the term part is used solely to denote a potential distinction between different objectinternal structural components, without implications concerning the relevant geometric property of the component (e.g., surface or volumetric shape primitive).

2. Vecera et al. (2000) attempted to address this issue by using displays of two multipart objects; not only did they replicate the part-based effect in their first experiment, but they also showed an object-based effect. The task, however, still required participants to explicitly direct attention to particular parts of objects in the stimulus displays.

3. There were 45 trials for each of the six cue-target locations where the cue appeared in the middle of the L shape (total of 270 trials). In addition, there were 35 trials for each cue-target location where the cue appeared either at the far end of the long arm or on the short arm of the object (total of 210 trials). Neither of these filler cue-target locations was of theoretical interest and were therefore excluded from the analyses.

4. We also conducted further analyses based on a calculation of IOR using other baselines for the three within-object conditions: (1) Condition 1 minus Condition 4; (2) Condition 2 minus Filler 1; (3) Condition 3 minus Filler 2. Planned comparisons on the resulting IOR effects between the critical conditions ( 2 and 3 ) showed a significant difference in the segmented displays $(12 \mathrm{msec})[t(9)=-2.3, p<.001]$ but not in the unsegmented displays $(7 \mathrm{msec})[t(9)=0.6, \mathrm{n} . \mathrm{s}$. $]$. These results show that using different baselines to calculate IOR does not change the pattern of object-based IOR modulation.

5. This finding rules out the possibility that responding to targets at either end of the object is more difficult than responding to targets in the middle of the object. More importantly, the finding that segmentation itself does not affect RTs establishes that the slower RTs for the same object/different part relative to the same part/different location condition were not due to a greater difficulty in detecting targets on smaller objects or parts of objects.

(Manuscript received April 10, 2001; revision accepted for publication March 20, 2002.) 